\title{
FSI methods for seismic analysis of sloshing tank problems
}

\author{
Zuhal OzDemir $^{1,2, a}$, Mhamed Souli $^{2}$ And Yasin M. Fahjan ${ }^{3}$ \\ 1 Bogazici University, Kandilli Observatory, Cengelkoy, Istanbul, Turkey \\ 2 Université de Lille, Laboratoire de Mécanique de Lille, CNRS 8107, Bd Paul Langevin, Villeneuve d'Ascq, France \\ 3 Gebze Institute of Technology (GYTE), Cayirova Campus, Kocaeli, Turkey
}

Received 5 May 2009, Accepted 13 December 2009

\begin{abstract}
The long-period components in earthquake ground motions, which attenuate gradually with distance, can induce sloshing waves in the liquid containment tanks although they are located far away from the seismic source. The resulting sloshing waves generate additional forces impacting the wall and roof of the tanks and may cause extensive damage on the tank structure. Numerous examples of tank damages due to sloshing of fluid have been observed during many earthquakes. Nevertheless, the effect of sloshing is usually primitively considered in most of the seismic design codes of tanks. On the other hand, the derivation of an analytical solution for the sloshing response of a liquid storage tank subjected to harmonic excitation includes many assumptions and simplifications. Most of the analytical solutions in the recent literature assumed the containing liquid to be invicid, incompressible and irrotational, and the tank structure to be an isotropic elastic plate with uniform stiffness, mass and thickness. Even though, experimental works are necessary to study the actual behavior of the system, they are time consuming, very costly and performed only for specific boundary and excitation conditions. However, appropriate numerical simulation using fluid structure interaction techniques can be used to predict the hydrodynamic forces due to the high-speed impacts of sloshing liquid on a tank wall and roof. These simulations can reduce the number of experimental tests. The nonlinear finite element techniques with either Lagrangian and/or Eulerian formulations may be employed as a numerical method to model sloshing problems. But, most of the Lagrangian formulations used to solve such problems have failed due to high mesh distortion of the fluid. The arbitrary Lagrangian Eulerian techniques are capable of keeping mesh integrity during the motion of the tank. In this study, an explicit nonlinear finite element analysis method with ALE algorithm is developed and sloshing phenomenon is analyzed. The analysis capabilities of the method are explained on a technical level. Although, the developed numerical procedure is applicable to deformable structures, the accuracy of the method is validated with the existing analytical formulation derived from potential flow theory as well as the experimental data carried out on rigid tanks when subjected to harmonic and earthquake ground motions. High consistency between numerical and experimental results in terms of peak level timing, shape and amplitude of sloshing waves is obtained not only for non-resonant excitation but also for resonant frequency motion.
\end{abstract}

Key words: Fluid-structure interaction / arbitrary Lagrangian-Eulerian algorithm (ALE) / sloshing / earthquake / tanks

Résumé - Méthodes sismiques FSI pour l'analyse des problèmes de ballottement dans les réservoirs. Les réservoirs pleins ou partiellement remplis de liquide sont vulnérables aux secousses sismiques. Selon l'amplitude et la durée des secousses sismiques, le ballottement du fluide sur les parois et le couvercle des réservoirs, peut provoquer des dommages considérables dans les zones de stockage même situées assez loin de la source du séisme. Dans les zones sismiques, de nombreux exemples d'endommagement dus au ballottement de fluide dans les réservoirs ont été observés lors d'un séisme. Néanmoins, l'effet de ballottement est généralement traité par des méthodes empiriques dans la plupart des codes de validation et de conception sismique des réservoirs. D'autre part, l'élaboration d'une solution analytique pour le ballottement dans le cas d'un réservoir de stockage de liquide soumis à une excitation harmonique comprend de nombreuses hypothèses et simplifications. La plupart des solutions analytiques dans la littérature considère que le fluide est non visqueux incompressible et irrationnel, et le réservoir une structure élastique isotrope ayant une rigidité et une épaisseur uniformes. Même si des études expérimentales

a Corresponding author: ozdemirzuhal@yahoo.com 


\begin{abstract}
sont nécessaires pour étudier le comportement réel du système, elles sont longues, très coûteuses et réalisées uniquement sous certaines conditions de chargement. En outre, des techniques numériques d'interaction fluide structure peuvent être un outil efficace pour l'analyse des réponses du système excitations au cours d'un séisme. Ces simulations peuvent réduire le nombre de tests expérimentaux nécessaires pour l'analyse du comportement sismique des réservoirs. La méthode des éléments-finis en formulation eulerienne ou ALE peut être utilisée pour la modélisation des forces hydrodynamiques dues au ballottement du fluide sur les parois du réservoir et du couvercle. En raison des fortes distorsions du maillage fluide, les techniques de mouvement de maillage sont mises en œuvre et détaillées dans cet article. Pour valider les méthodes développées dans cet article, les résultats numériques obtenus sont comparés aux résultats expérimentaux et théoriques provenant de la littérature. Dans les approches théoriques, certaines hypothèses pouvant simplifier l'étude théorique sont prises en compte. Le fluide est considéré comme un fluide potentiel, la structure rigide et le mouvement imposé est un mouvement harmonique. En terme de valeurs maximales de pression, d'amplitude des ondes générées par le mouvement du fluide, les résultats numériques développés dans ce papier sont consistants et sont comparables à ceux obtenus par les méthodes théoriques et empiriques utilisées pour les codes de validation et de conception.
\end{abstract}

Mots clés : Interaction fluide structure / algorithme ALE / ballottement / séisme / réservoirs

\section{Introduction}

Sloshing in liquid containment tanks, generally, is not a negligible phenomenon even at very small amplitude earthquake excitations because of the large amount of the liquid content and low stiffness of the tank shell. This phenomenon may represent violent fluid motion with strongly non-linear manner involving high-speed impacts on tank walls and roof in oblique sense, breaking waves, and liquid droplet formation. The amplitude of the sloshing, in general, depends on the nature, amplitude and frequency of the earthquake motion, liquid fill level, liquid properties and tank geometry. When the frequency of the motion is close to one of the natural frequencies of liquid sloshing, large amplitudes sloshing waves can be expected. High hydrodynamic forces generated by sloshing waves on tank affect the overall response of the tank and cause a complicated fluid-structure interaction problem between two materials.

Although sloshing is a difficult mathematical problem to solve analytically, early treatments of this problem were carried out with analytical methods established on potential flow theory disregarding all viscous effects. In these studies, the irrotational motion of invicid and incompressible fluid inside the rigid container was represented with Laplace equation. The first approximate solution for a rigid cylindrical tank under horizontal motion was provided by Jacobsen [1] on the basis of a closedform solution of the Laplace equation that satisfies specified boundary conditions. Housner [2-4] used an approximate method idealizing the liquid as being constraint by rigid membranes to compute the hydrodynamic pressures developed in a rigid cylinder and rectangular fluid container subjected to horizontal accelerations. Veletsos and Yang [5] split hydrodynamic effects obtained from Laplace equation in two parts namely the "impulsive" and the "convective" motions. Faltinsen [6] derived a linear analytical solution for liquid sloshing in a horizontally excited 2D rectangular tank considering damping due to viscous effects. Fischera and Rammerstorferb [7] investigated analytically the overall effect of pressure generated by interaction forces between sloshing and the wall motion modifying the free surface boundary conditions.

Since analytical methods are restricted to small motions of the sloshing fluid, the numerical solution algorithms, which take into account accurately the all source of nonlinearity of the sloshing problem have been developed over the years. In these methods, fluid motion inside the container have been represented with either Laplace, Euler, wave or Navier-Stokes equations which have been solved employing boundary element method (BEM), finite difference method (FDM), the finite-element method (FEM). Lay [8] developed a numerical model for the seismic analysis of tanks with single and double curvatures, which was achieved by transforming the boundary-element equations of the incompressible and inviscid fluid region governed by the Laplace equation into an equivalent finite-element mass matrix, which was then combined with the shell finite-element equations of motion. El-Zeiny [9] formulated fluid motion with Laplace equation and developed a finite-element program, which uses an updated Eulerian-Lagrangian description of the liquid-structure interface in order to enforce compatibility between structure and liquid elements, to analyze the large amplitude liquid sloshing and nonlinear liquid-structure interaction. Chen and Chiang [10] employed time independent finite difference method to study sloshing inside 2D tank with rigid walls solving incompressible Euler equation under fully nonlinear kinematic free-surface condition. This study was extended by Chen [11] adding fluid viscous effects. Souli et al. [12] and Souli and Zolesio [13] developed a procedure for fluid-structure interaction problems based on Arbitrary Lagrangian Eulerian (ALE) algorithm of finite-element method and validated the applicability of the procedure for sloshing problems. For low magnitude loading, the sloshing problem can be solved using incompressible Navier Stokes equations with an implicit or explicit coupling to the structure. Longatte et al. $[14,15]$ applied this methodology where the fluid structure interface is 
described by the mesh nodes at the interface. For large amplitude loading that generates large structure deformation and fluid high mesh distortion, a coupling between Lagrangian structure and Eulerian fluid formulation can be used as detailed by Aquelet et al. [16]. The sloshing behaviors of fluid in 3D rigid cylindrical and rectangular tanks subjected to horizontal oscillations were addressed with a numerical and experimental study by Chen et al. [17]. Liu and Lin [18] adopted finite difference method which solves Navier-Stokes equations to study 2D and $3 \mathrm{D}$ viscous and inviscid liquid sloshing in rectangular tanks and verified the results with the linear analytical solution and experimental data. Mitra et al. [19] used finite element method to solve wave equation to quantify liquid sloshing in partially filled 2D rigid annular, horizontal cylindrical and trapezoidal containers.

In order to validate theoretical and numerical solutions, experiments are the very powerful source to obtain information about sloshing and tank response due to external loadings. Several laboratory measurements have been conducted to quantify sloshing wave height, hydrodynamic pressure and shell stresses. Kana [20] measured wall stresses of cylindrical flexible tank induced by sloshing and inertial loads experimentally. Manos [21] carried out experiment to determine impulsive mode frequencies and base-overturning moments of broad and tall tanks. Tanaka et al. [22] conducted dynamic tests on small and large scale models under earthquake loading in order to investigate elephant foot buckling and side slipping behavior of cylindrical tanks. The sloshing wave heights in $2 \mathrm{D}$ and $3 \mathrm{D}$ rectangular tanks subjected to external loads were measured experimentally by Liu and Lin [18]. The more extensive literature review and detailed investigation of sloshing problem from basic theory to advanced analytical and experimental studies can be found in the work of Ibrahim [23].

In the present study, a new computational procedure utilizing ALE algorithm is developed to solve fluid-structure interaction problems with or without free surface. The procedure used in this numerical method is explained in short. The governing equations for fluid and structure and their solution methodologies are clarified. The numerical approaches in the algorithm used to track the free surface and advection of fluid material across element boundaries are reviewed. The present numerical method is applied for the analysis of the sloshing in a small-scale tank subjected to external excitations. The accuracy of the numerical method is validated on rigid tank models using existing analytical formulation derived from potential flow theory as well as the experimental data in terms of wave height of the liquid free surface.

\section{Kinematical description of ALE algorithm}

The conventional finite element procedure for the solution of engineering and academic problems including fluid-structure interaction effects are usually based on a purely Lagrangian algorithm because of easy implementation of this algorithm. But, these problems generally involve large deformations and construction of new free surfaces and can not be handled by the same Lagrangian mesh during the entire simulation since severely distorted elements have low accuracy and their stable time step sizes are small for explicit time integration algorithms to continue the simulation [24]. In this case, a new mesh must be generated and the old solution must be transferred from the old mesh onto the new mesh. This remeshing process can be achieved by a rezoning method where automatic mesh generators are called internally to create a new mesh with a new topology [25]. In the rezoning methods, the dependent variables, such as velocity, pressure, internal energy, stress components and plastic strain, are updated on the new mesh by using a remap algorithm. The other alternative to construct undistorted mesh is to use ALE algorithm which control mesh geometry independently from material geometry. Unlike a rezoning method, the topology of the mesh is fixed in an ALE algorithm where only the mesh nodes are relocated to obtain a homogeneous and undistorted mesh. The accuracy of an ALE calculation is often superior to the accuracy of a rezoned calculation because the algorithms used to remap the solution from the distorted to the undistorted mesh is second order accurate for the ALE formulation when using second order advection algorithms, while the algorithm for the remap in the rezoning is only first order accurate.

The Arbitrary Lagrangian-Eulerian (ALE) algorithm was developed in an attempt to combine the advantages of the Lagrangian and Eulerian kinematical descriptions, while minimizing their respective drawbacks as far as possible. An ALE algorithm contains both pure Lagrangian and pure Eulerian descriptions. This algorithm is based on the arbitrary movement of a referential domain, which is introduced as a third domain in addition to the common material (Lagrangian) domain and spatial (Eulerian) domain [26]. In this referential domain, which will later on correspond to the finite-element mesh, the problem is formulated. The arbitrary movement of the reference frame, accompanied of course by a good "mesh moving algorithm", enables us to rather conveniently deal with moving boundaries, free surfaces, large deformations, and interface contact problems.

Let $\Omega_{X}$ represents the material (Lagrangian) domain, $\Omega_{x}$ represents the spatial (Eulerian) domain and $\Omega_{\xi}$ represents the referential domain. In order to describe material and mesh motions, mappings must be defined between these three domains. The mapping from $\Omega_{X}$ to $\Omega_{x}$ defines the particle motion in the spatial domain, whereas the mapping from $\Omega_{\xi}$ to $\Omega_{x}$ describes mesh motion in the spatial domain. Through the mapping from $\Omega_{\xi}$ to $\Omega_{X}$ results in convection (Fig. 1).

Referencing to arbitrary Lagrangian Eulerian algorithm described by Hughes et al. [26], total material time derivative of a physical property, $\phi$, at fixed initial (Lagrangian) coordinate is decomposed into a local derivative at fixed referential coordinate, describing the change of $\phi$ with respect to this referential (ALE) configuration, and in a convective part that considers the relative 


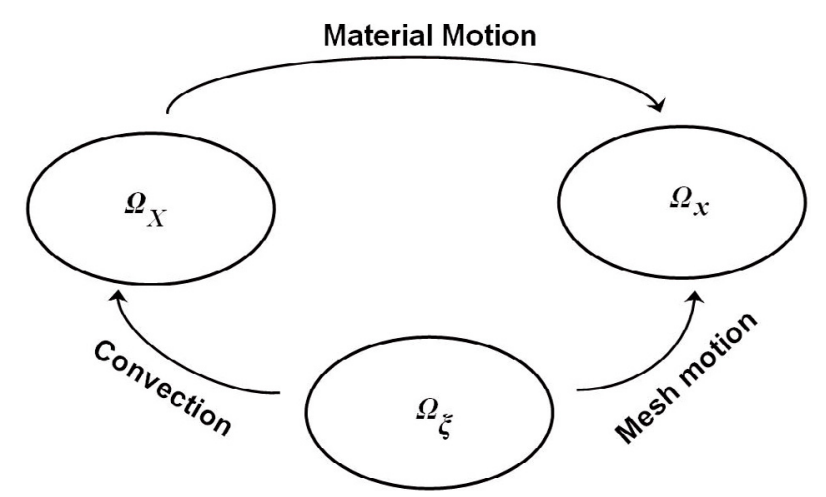

Fig. 1. Material, spatial and referential domains.

motion of the referential (ALE) configuration with respect to the initial configuration. This relation can be formulized as follows:

$$
\frac{\mathrm{d} \phi(\vec{X}, t)}{\mathrm{d} t}=\frac{\partial \phi(\vec{\xi}, t)}{\partial t}+\vec{c} \operatorname{grad} \phi(\vec{\xi}, t)
$$

where $\vec{X}$ is the Lagrangian coordinate, $\vec{\xi}$ is the reference (ALE) coordinate, $\vec{x}$ is the Eulerian coordinate, $\vec{c}$ is the convective velocity which is the relative motion of the velocity of the fluid particles $\vec{\nu}$, and to the velocity of the reference coordinate (mesh) $\vec{u}$ :

$$
\vec{c}=\vec{v}-\vec{u}
$$

It should be remarked that both Lagrangian and Eulerian algorithms are a particular case of ALE algorithm. The Eulerian algorithm is derived by assuming that the reference coordinate (mesh) velocity is zero, therefore the relative velocity between the material and the reference configuration is the material velocity. The Lagrangian algorithm is obtained when the reference coordinate (mesh) velocity equals to material velocity which means convective velocity is null.

\section{Governing equations for fluid and structure in ALE algorithm}

In order to compute interaction forces between fluid and structure, balance equations of both materials are defined with one of the kinematical description of continuum mechanics (Lagrangian Eulerian and arbitrary Lagrangian Eulerian) and solved utilizing boundary conditions and constitutive relations of materials. The balance equations for fluid and structure are formulated with three conservation equations of mass, momentum and energy. Although fluid and structure are governed by the same balance equations, the constitutive equations, which describe material behavior and relate stress to a measure of deformation, of two materials are different. The conservation equations of mass momentum and energy of fluid are formulated with Navier-Stokes equations which are solved to compute the fluid properties such as density, velocity and pressure.
Since material passes between elements of the computational mesh in ALE algorithm, the density has to be balanced explicitly to account for the conservation of mass during the non-Lagrangian deformation. The equation of mass conservation for a compressible Newtonian fluid in the ALE form is given by:

$$
\frac{\partial \rho}{\partial t}+\rho \operatorname{div}(\vec{v})+(\vec{v}-\vec{u}) \operatorname{grad}(\rho)=0
$$

The conservation of momentum equation is expressed as:

$$
\rho \frac{\partial \vec{v}}{\partial t}+\rho(\vec{v}-\vec{u}) \operatorname{grad}(\vec{v})=\operatorname{div}(\sigma)+\vec{F}
$$

The conservation of total energy equation for the ALE algorithm can be written by:

$$
\rho \frac{\partial e}{\partial t}+\rho(\vec{v}-\vec{u}) \operatorname{grad}(e)=\sigma: \dot{\varepsilon}+\vec{F} \vec{v}
$$

where $\rho$ is the density, $\dot{\varepsilon}$ is the strain rate tensor, $e$ is the internal energy per unit volume, $\vec{F}$ is the body force, $t$ is time. Total Cauchy stress, $\sigma$, is defined as the summation of the pressure and deviatoric terms:

$$
\sigma=-P \mathrm{Id}+2 \mu \stackrel{\bullet}{\varepsilon}
$$

where Id is the identity tensor, $P$ is the pressure and $\mu$ is the dynamic viscosity of the fluid. The pressure term is obtained from equation of state which relies pressure to density and internal energy, whereas constitutive equations are used to compute deviatoric part.

The terms in Equations (3)-(5) related to the convective velocity are usually referred to as the advection terms, and accounts for the transport of the material past the mesh. These additional terms in the equations make solving the ALE equations much more difficult numerically than the Lagrangian equations, where the relative velocity $\vec{c}$ is zero. In the Eulerian form of Navier-Stokes equations, although the velocity of the reference coordinate (mesh), $\vec{u}$, is zero, the Navier-Stokes Equations (Eqs. (3)-(5)) are not simplified, only the remeshing and smoothing processes are eliminated.

Although any of the Lagrangian, Eulerian or ALE algorithms can be used to model the fluid motion for fluid-structure interaction problems, the structure is always discretised with Lagrangian approach. Since in Lagrangian algorithm computational mesh nodes always follow the associated points of the material domain during motion, advection term does not exist. Mass conservation equation for structure is satisfied spontaneously. Momentum and energy conservation equations for Lagrangian algorithm can be given as follows respectively:

$$
\begin{aligned}
& \rho \frac{\mathrm{d} \vec{v}}{\mathrm{~d} t}=\operatorname{div}(\sigma)+\vec{F} \\
& \rho \frac{\mathrm{d} e}{\mathrm{~d} t}=\sigma: \dot{\varepsilon}+\vec{F} \vec{v}
\end{aligned}
$$

where $\sigma$ is the total Cauchy stress given by:

$$
\sigma=-P \operatorname{Id}+2 G \varepsilon
$$


In this formulation $G$ represents shear modulus and, $\varepsilon$ symbolizes shear strain.

\section{Solution of Navier-Stokes equations in ALE form}

There are two ways to solve Navier-Stokes equations in ALE form. In the first method, these equations with fully coupled form are solved by integrating forward in time which is very time consuming $[16,27,28]$. The more widely favored approach is to employ operator split method which treats these equations in two distinct phases namely, Lagrangian and advection phases, in each time step. In the first phase all advective effects are neglected and the reference system is forced to follow the material flow as a Lagrangian manner. The physical material deformations are determined according to the equilibrium equations of Lagrangian phase (Eqs. (7) and (8)) and the constitutive equations. The changes in velocity, displacement, pressure and internal energy due to the internal and external forces are calculated. Transport of material between elements is considered in advection phase. All the physics of the problem is carried out in the Lagrangian phase while the advection phase is purely digital.

From a discretization point of view, Equation (7) is computed using one integration point for efficiency and in order to eliminate locking [29]. The zero energy modes are controlled with an hourglass viscosity [30]. Although the continuity equation in partial differential equation form can be used to obtain current density in Lagrangian phase, its integrated form (Eq. (10)) which is more accurate is employed [24]:

$$
\rho J=\rho_{0}
$$

where $\rho$ is the current density, $\rho_{0}$ is the initial density and $J$ is the volumetric strain given by the Jacobian:

$$
J=\operatorname{det}\left(\frac{\partial x_{i}}{\partial X_{j}}\right)
$$

Central difference method, which is derived from Taylor series expansion, is employed to advance the position of the mesh in time using an explicit method in which the solution progresses without any iteration between consecutive time steps. In order to have a second order accurate scheme in time, the velocity must be staggered with respect to the displacement:

$$
x^{n+1}=x^{n}+\Delta t u^{n+1 / 2}
$$

The internal nodal forces which are a function of stresses and external forces associated with body forces and boundary conditions (boundary forces, non-reflection boundary forces and contact forces) are used to update the velocity in time:

$$
u^{n+1 / 2}=u^{n-1 / 2}+\Delta t^{n} M^{-1}\left(F_{\text {ext }}^{n}+F_{\text {int }}^{n}\right)
$$

where $F_{\text {int }}$ is the internal force vector and $F_{\text {ext }}$ is the external force vector and $M$ is the mass matrix diagonalized. For each element of the mesh, the internal force is computed as follows:

$$
F_{\text {int }}=\sum_{k=1}^{\text {Nelem }} \int_{k} B^{t} \sigma \mathrm{d} v
$$

where $B$ is the gradient matrix and $N_{\text {elem }}$ is the number of elements.

Since the central difference method is explicit, a finite stable time step size which is necessary for a sound wave to cross an element in the mesh must be below a critical value to provide numerical stability (courant condition [25]):

$$
\Delta t \leq \frac{\Delta x}{c}
$$

where $\Delta x$ is the length of the smallest element in the mesh, $\vec{c}$ is the speed of sound in the material [31]. For a solid material, the speed of sound is:

$$
\begin{aligned}
c^{2} & =\frac{\frac{4}{3} G+k}{\rho_{0}} \\
k & =\rho_{0} \frac{\partial P}{\partial \rho}+\frac{P}{\rho} \frac{\partial P}{\partial e}
\end{aligned}
$$

where $\rho$ is the material density, $G$ is the shear modulus, and $P(\rho, e)$ is the equation of state. In Equation (17), the second term on the right hand side accounts for the stiffening effect due to the increase of internal energy as the material is compressed. For a fluid material, $k=\rho_{0} c^{2}$ in which $\rho_{0}$ is the mass density and $c$ is the sound velocity. The viscosity of the fluid material is generally ignored in the calculation of the speed of sound. For sloshing problems the pressure component of stress is much greater than the deviatoric part of the stress due to the viscosity of the fluid, and the deviatoric stress is sometimes ignored.

ALE algorithm allows the finite element mesh to contain more than one material within the same element as well as each element can be restricted to contain a single material. For the single material ALE (SALE) case, the material interfaces are resolved directly by the finiteelement mesh as in the Lagrangian sense and no material fluxes over element boundaries have to be considered during the advection process. For the large deformation problems e.g. sloshing problems, it is advantageous to apply multi-material ALE (MMALE) approach, where the material boundaries can run freely through the finite-element discretization $[25,29]$. For the multi-material ALE case, before the advection phase of operator split method, an interface-tracking algorithm is performed in order to compute accurately the material interfaces in the ALE elements containing several fluid materials.

The most popular methods to track interfaces between multi-materials are the volume of fluid method (VOF) [32], which was originally developed for the finite volume method (FVM) and the marker and cell (MAC) method $[33,34]$. 
The MAC method involves Eulerian flow calculation and Lagrangian particle movement. The velocity of the markers is found first by locating the fluid cell containing the particle and taking the average velocities of the cell nodes (the average is based on the finite element particles in the fluid cell). The particle cells have small inertia and tend to follow the fluid flow. However, the MAC method becomes complicated if the interfaces become highly distorted or if the geometry is complex.

The tracking of the material deformations can be performed by the VOF (Volume of Fluid) method or the Young method [35] which is attractive for solving a broad range of non-linear problems in fluid and solid mechanics such as, sloshing and explosion applications [34, 36], because it allows arbitrary large deformations and enables free surfaces to evolve. Moreover, the Lagrangian phase of the VOF method is easily implemented in an explicit ALE finite-element method. In this method, different material occurrences are considered by their respective volume fractions on the element level. For multi-material elements, the volume fraction of one fluid satisfies:

$$
V_{\mathrm{f}} \leq 1
$$

The total stress by $\sigma$ is weighed by volume fraction to get the fluid stress fields:

$$
\sigma_{\mathrm{f}}=\sigma V_{\mathrm{f}}
$$

The Young or volume of fluid (VOF) method is originally developed to track an interface in elements containing two materials for two-dimensional problems. This method is adapted in this paper for the three-dimensional problems. The VOF method described in this paper is based on flux transport between element faces. The transport is based on the interface between the two materials in the element. This interface is constructed by using the gradient of the nodal volume fraction of materials.

The material layout is described solely by the volume fraction repartition of the fluid material in the ALE elements. Specifically, a straight line using the simple linear interface calculation (SLIC) technique of Woodward and Collela [38] approximates the interface in the cell. Interfaces are initially drawn parallel to the element faces. Then nodal volume fraction $f$ is computed to each node based on the fraction volumes of elements that share the same node. This nodal volume fraction repartition determines the slope of the material interface inside the element. The normal vector to the interface inside the element is defined by

$$
\vec{n}=\frac{\overrightarrow{\operatorname{grad}} f}{|\overrightarrow{\operatorname{grad}} f|}
$$

The position of the interface is then oriented by the normal $\vec{n}$ so that it divides the element into two volumes, which correctly matches the element volume fraction (Fig. 2). The interface position is used to calculate the volume of the fluid flowing across cell sides. As the $X$ advection, $Y$-advection and $Z$-advection are calculated in

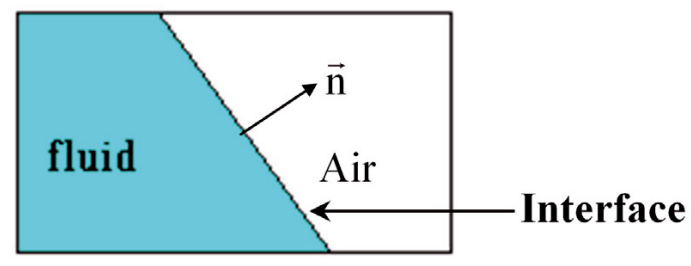

Fig. 2. Interface between two materials, air and fluid.

separate steps, it is sufficient to consider the flow across one side only.

For the single material and voided element case, the same procedure is applied. For voided elements, the stress is zero. In the computational process, the elements loop goes only through elements that are not voided. For free surface problems, the elements that are partially filled $\left(V_{\mathrm{f}}<1\right)$ define the free surface. The location of free surface in a sloshing problem is defined with interfacetracking algorithm.

Tracking of interfaces of different materials in an element is followed by an advection phase in which the solution on the displaced mesh at the end of the Lagrangian phase is mapped into its original position for an Eulerian formulation or arbitrary position for an ALE formulation. In this phase, transport of history state variables: mass, internal energy and momentum across cell boundaries are computed. The transport equations for the advection phase are

$$
\begin{gathered}
\frac{\partial \phi}{\partial t}+c \operatorname{grad}(\phi)=0 \\
\phi(\vec{x}, 0)=\phi_{0}(x)
\end{gathered}
$$

The transport equation (Eq. (21)) with initial condition (Eq. (22)) obtained from Lagrangian cycle of operator split method is applied for being the density, momentum per unit mass, and energy per unit mass:

$$
\phi: \rho, \rho v, \rho e
$$

where $\vec{c}$ is the convective velocity defined in Equation (2). In the advection phase, the hyperbolic transport equation (Eq. (21)) is solved successively for the conservative variables mass, momentum and energy (Eq. (23)) with initial condition, $\Phi_{0}(x)$, which is the solution from Equations (7) and (8) of Lagrangian phase at the current time. In equation (21), the time $t$ is a fictitious time. In this paper, time step is not updated when solving for the transport equation. Lagrangian and advection phases are carried out within the same time step. There are different ways of splitting the Navier-Stokes problems. In some split methods, each of the Lagrangian phase and advection phase is solved successively for half time step. For the solution of Equation (21), the Donor Cell algorithm, a first order upwind method and the second-order Van Leer algorithm [39] can be used. These algorithms have already been described in detail by Benson [25], Benson [40] and Souli et al. [12]. 
In this study, momentum is advected instead of velocity to guarantee the conservation of momentum, and the velocities are calculated by dividing the values of the momentum at the nodes by the associated masses. The momentum advection is carried out after the element centered mass advection because the new masses are needed to calculate the new velocities from the momentum. To prevent distribution of momentum from nodes to elements during the advection and from elements to nodes during nodal velocity calculations, the momentum advection is done only through the nodes. This procedure is carried out with a staggered mesh which is constructed with respect to the original mesh so that the original mesh centroids become the nodes of the new mesh [25]. Amsden and Hirt [41] developed the first code to construct a staggered mesh for the momentum advection and the basic idea is still in common use.

A cell centered advection algorithm is applied to the staggered mesh for the momentum advection. The data necessary for the advection algorithm are the cell volume before and after the Lagrangian phase, nodal velocities, nodal masses and fluxes between cells. All the data are ready on the staggered mesh except for the fluxes. The new flux values on the staggered mesh are defined using a regular distribution of the fluxes from the original mesh element faces to the new element faces. Once the new flux on the staggered mesh is computed, the momentum advection is performed according to the following algorithm:

$$
V^{+} M^{+}=V^{-} M^{-}+\sum_{j=1}^{N \text { bedges }} V_{j}^{-} M_{j}^{-}
$$

where the superscripts "-" and "+" refer to the solution values before and after the transport. Values that are subscripted by $j$ refer to the boundaries of the elements, through which the material flow, and the $V_{j}^{-}$ are the fluxes transported through the adjacent elements, these fluxes are computed using the staggered mesh. The flux is positive if the element received material and negative if the element is loosing material.

For the energy advection either internal energy or total energy can be used. If internal energy is advected, total energy can not be conserved and usually decreases with time because momentum advection does not conserve kinetic energy. The decrease in kinetic energy corresponds to the diffusion of velocity through the mesh. In order to solve this problem total energy is advected and the internal energy in an element is calculated as the difference between the total energy and kinetic energy.

The overall flow of the calculations in the ALE method presented in this paper can be summarized as follows:

1. A Lagrangian step is performed first:

(1.1) A new time step size is calculated based on the speed of sound through each of the elements and their geometry.

$$
\Delta t^{n} \leq \frac{\Delta x}{c}
$$

(1.2) Strain rate, $\dot{\varepsilon}$, is computed using nodal velocity of elements.

$$
\dot{\varepsilon}^{n}=\frac{1}{2}\left(u_{i, j}^{n}+u_{j, i}^{n}\right)
$$

(1.3) Deviatoric stress component is computed using material model for fluid.

(1.4) Internal energy is updated using energy equation.

(1.5) Pressure is computed using equation of state for fluid.

(1.6) Internal forces are computed (Eq. (14)).

(1.7) Acceleration, velocity, and displacement are calculated using explicit time integration.

End of Lagrangian step

2. An advection algorithm is performed next.

(2.1) New positions of the nodes are computed for the ALE algorithm.

(2.2) Advection of the conservative variables: mass, momentum, and energy, using a transport equation (Eq. (21)).

End of advection step

\section{Analytical formulation for sloshing in rigid tank subjected to harmonic motion}

The analytical solution given in this paper regards tank problem with sloshing liquid subjected to periodic harmonic motion as an initial boundary-value problem. A two-dimensional rigid rectangular tank system with a length of $2 a$ is partially filled with liquid to a height of $h$. A Cartesian co-ordinate system $(x, y)$ with the origin at the centre of the free surface of the fluid is used. The forced excitation of tank is taken as a horizontal harmonic displacement movement of the base:

$$
d_{\mathrm{e}}=-D \sin (\varpi t)
$$

for $t>0$. By neglecting the effects of liquid compressibility, viscosity and surface tension, the irrotational motion of fluid inside the tank can be formulized with Laplace equation as:

$$
\nabla^{2} \Phi=0
$$

where $\Phi$ represents velocity potential function of fluid. In order to simply the solution, the total velocity potential function is split into a disturbance potential function due to internal sloshing flow, $\Phi_{1}$, and a potential function, $\Phi_{\mathrm{C}}$, which defines the motion of the tank, as,

$$
\Phi=\Phi_{1}+\Phi_{\mathrm{c}}
$$

The corresponding essential boundary conditions used for the solution of Laplace equation are linearized assuming the boundaries are rigid. The velocity boundary conditions that specify the liquid motion along the tank bottom and wall are defined as following:

$$
\frac{\partial \Phi_{1}}{\partial r_{n}}=0 \text { on the wetted boundary }
$$


where $r_{n}$ is surface normal. Nonlinear effects are ignored assuming the wave amplitudes are very small in comparison with the wavelengths and depths. Therefore, kinematic and dynamic boundary conditions are implemented at the undisturbed free surface of the liquid. Kinematic free surface boundary condition which states a fluid particle on the free surface at some time will always remain on the free surface and dynamic free surface boundary condition which requires the pressure on the free surface is zero are given, respectively, as follows:

$$
\begin{gathered}
\frac{\partial \eta}{\partial t}=\frac{\partial \Phi}{\partial y} \text { on the boundary } y=0 \\
\frac{\partial \Phi}{\partial t}+g \eta=0 \quad \text { on the boundary } y=0
\end{gathered}
$$

where $\eta$ is free surface displacement, measured from the undisturbed liquid surface at equilibrium and $g$ is the acceleration of gravity. The combination of kinematic and dynamic boundary conditions at the free surface in terms $\Phi_{1}$ gives:

$$
\frac{\partial^{2} \Phi_{1}}{\partial t^{2}}+g \frac{\partial \Phi_{1}}{\partial y}=-\omega^{3} D \cos (\omega t) x
$$

Under boundary conditions (Eqs. (30) and (33)) the solution of Laplace (Eq. (28)) gives the following expression for velocity potential of fluid domain inside the tank [6]:

$$
\begin{aligned}
& \Phi(x, y, t)=\sum_{n=0}^{\infty} \sin \left\{\frac{(2 n+1) \pi}{2 a} x\right\} \cosh \\
& \times\left\{\frac{(2 n+1) \pi}{2 a}(y+h)\right\}\left(A_{n} \cos \left(\varpi_{n} t\right)+C_{n} \cos (\varpi t)\right) \\
& -\varpi D \cos (\varpi t) x
\end{aligned}
$$

where

$$
\begin{gathered}
A_{n}=-C_{n}-K_{n} / \varpi \\
C_{n}=\frac{\varpi K_{n}}{\varpi_{n}^{2}-\varpi^{2}} \\
K_{n}=-\frac{\varpi^{2} D}{\cosh \left\{\frac{(2 n+1) \pi}{2 a} h\right\}} \frac{2}{a}\left(\frac{2 a}{(2 n+1) \pi}\right)^{2}(-1)^{n}
\end{gathered}
$$

In the case of free vibration (i.e. if the r.h.s. of Eq. (33) is 0$)$, the circular frequencies $\omega_{n}$ of sloshing modes are calculated as following:

$$
\omega_{n}^{2}=g \frac{(2 n+1) \pi}{2 a} \tanh \left(\frac{(2 n+1) \pi}{2 a} h\right)
$$

where $n$ represents mode number. The free surface displacement, $\eta$, measured from the undisturbed liquid surface at equilibrium, and pressure, $p$, can be defined in terms of velocity potential function as follows:

$$
\begin{aligned}
p(x, y, t) & =-\rho \frac{\partial \Phi}{\partial t} \\
\eta(x, t) & =\frac{1}{g} \frac{\partial \Phi}{\partial t}
\end{aligned}
$$

where $\rho$ is the mass density of the liquid.

\section{Numerical simulations for sloshing in rigid tanks under horizontal excitations}

The validity of the numerical method presented in this paper for sloshing problems is verified with three different experimental studies under both harmonic and earthquake excitations in terms of free surface profile and wave height.

\subsection{Sloshing free surface profile under harmonic motion}

In order to demonstrate the applicability of the developed analysis code for sloshing problems, a series of experiments is performed at the shaking table laboratory of the Kandilli Observatory and Earthquake Research Institute of the Bogazici University. The sloshing event inside the experimental tank model is compared with the numerical one in terms of free surface profile.

A small scale shaking table, which can be controlled to introduce pulse, sinusoidal and random motions in one horizontal component, is used to simulate sinusoidal motion for sloshing experiment. Considering the dimensions, weight limit and the frequency ranges of the shaking table, a scaled model is built to investigate the fluid free surface profile under horizontal sinusoidal motion. Rectangular geometry is chosen for the scaled model to get rid of the effect of other horizontal dimension on sloshing phenomena. The scaled glass rectangular tank model is fabricated with a width of $0.15 \mathrm{~m}$, a breadth of $0.10 \mathrm{~m}$, total height of $0.35 \mathrm{~m}$ and thickness of $0.005 \mathrm{~m}$. Water is filled at a level of $0.15 \mathrm{~m}$ inside the model. The first three fundamental sloshing frequencies for this configuration are computed to be $2.28,3.23$ and $3.95 \mathrm{~Hz}$ from Equation (38). Two harmonic tests are conducted on the physical experimental setup applying sinusoidal motion with displacement amplitude of $0.005 \mathrm{~m}$ and frequencies of $2.7 \mathrm{~Hz}$ and $3.5 \mathrm{~Hz}$, which are non-resonant frequencies for this configuration.

Since the scaled model has very narrow breadth, and that the excitation is only in the width direction, it is modeled as $2 \mathrm{D}$ rectangular in shape in the numerical simulations. Rigid shell elements are used for tank itself and the Eulerian mesh for the fluid domain is constructed with ALE solid element. The Eulerian mesh of the model consists of 3724 solid elements with a typical edge length of $0.005 \mathrm{~m}$, and the Lagrangian mesh consists of 240 shell elements with a typical edge length of $0.005 \mathrm{~m}$. The model contains 8202 nodes. Water inside the container is modeled with incompressible and invisicid elastic fluid material $\left(\rho=1000 \mathrm{~kg} \cdot \mathrm{m}^{-3}\right)$. The same loading conditions of the experimental studies are applied to the numerical ones.

The free surface profiles of the rectangular tank model obtained from experimental and numerical studies are compared at an arbitrary instant for each loading cases. The comparison of results of two studies is shown in Figure 3 at time $1.25 \mathrm{~s}$ for the situation when the amplitude and frequency of the applied harmonic 

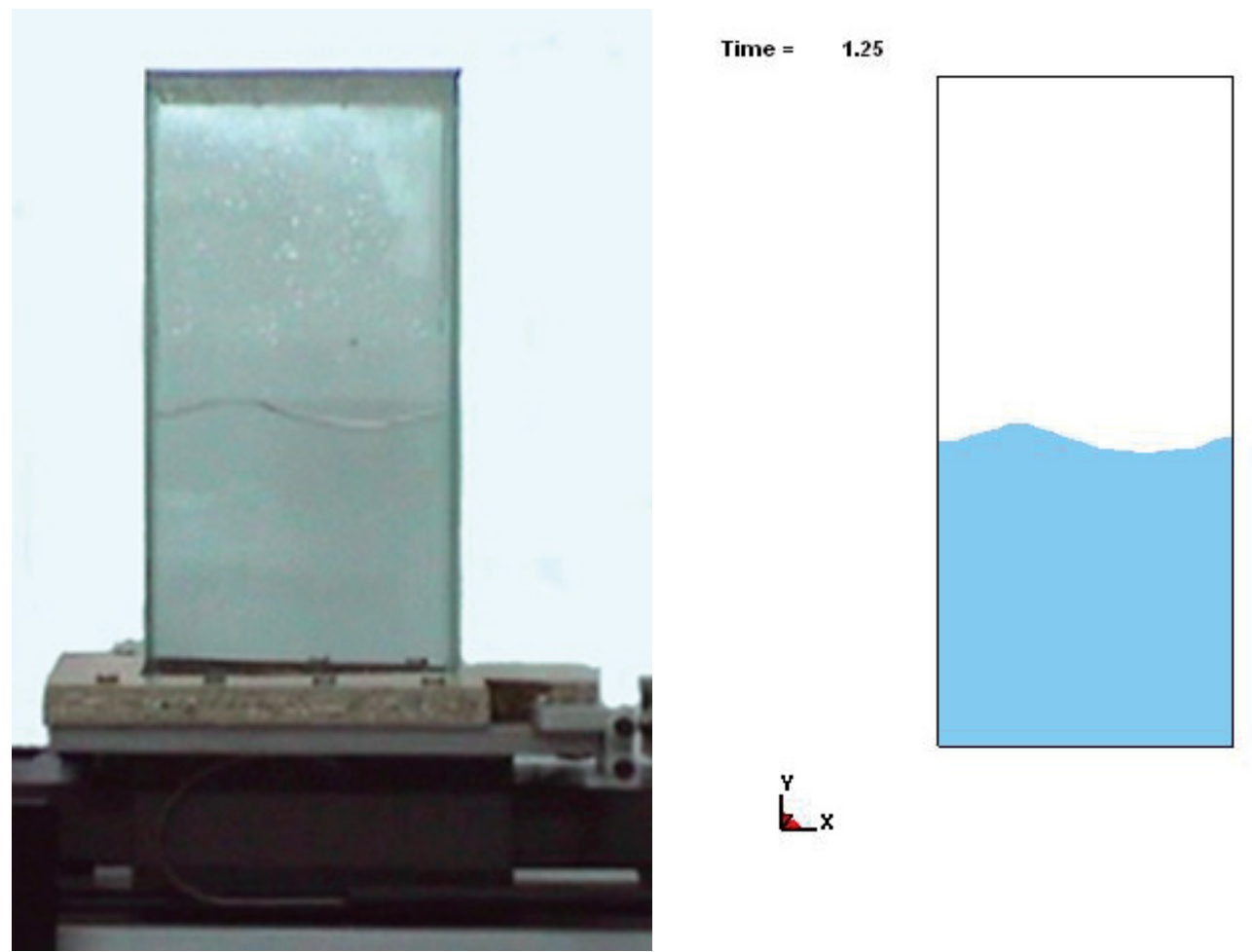

Fig. 3. Free surface profiles of experimental and numerical models under sinusoidal excitation with amplitude $0.005 \mathrm{~m}$ and frequency $2.7 \mathrm{~Hz}$.

base displacement are $0.005 \mathrm{~m}$ and $2.7 \mathrm{~Hz}$, respectively. Figure 3 indicates that the free surface profile obtained from experimental study perfectly matches with the numerical one. For the second loading case which has displacement amplitude of $0.005 \mathrm{~m}$ and frequency of $3.5 \mathrm{~Hz}$, free surface profiles of experimental and numerical studies are given in Figure 4 at time $0.76 \mathrm{~s}$. This figure obviously shows that two surface profiles are in fairly good agreement.

\subsection{Sloshing wave height under harmonic motion}

The sloshing event inside a 3D rigid tank subjected to harmonic motion is investigated with fully nonlinear fluidstructure interaction algorithm based on ALE approach implemented in this paper. Numerical simulation results in terms of displacement of free surface are compared with the corresponding results of analytical formulation given in Equation (34) and the shaking-table test results of Liu and Lin [18]. The same loading conditions and dimensions used in the experimental study are employed for the analytical and numerical models. Experimental data is used as reference solution for sloshing problem.

In the numerical study, a three-dimensional rigid rectangular tank with a width of $0.57 \mathrm{~m}$, breadth of $0.31 \mathrm{~m}$ and total height of $0.30 \mathrm{~m}$ is filled with water $(\rho=$ $1000 \mathrm{~kg} . \mathrm{m}^{-3}$ ) up to a height of $0.15 \mathrm{~m}$. The interior liquid is discretized with uniform mesh. The model is motionless at time $t=0$ and harmonic motion is applied as displacement. The time step size is $1 \times 10^{-4} \mathrm{~s}$ throughout the simulation.
Two loading cases as non-resonance and resonance are considered. In view of the first fundamental sloshing frequency which can be obtained from Equation (38) as $\omega_{0}=6.0578 \mathrm{rad} . \mathrm{s}^{-1}$, the excitation frequency of the first case was taken by $\omega=0.583 \omega_{0}$. The second loading is intended to simulate sloshing phenomenon under resonant frequency, therefore the excitation frequency is taken as the same as first fundamental frequency. The amplitudes of the horizontal harmonic excitations are $0.005 \mathrm{~m}$ for both cases. The time history response of free surface elevation is measured at three locations which were near left (i.e. $x=-0.265$ ) and right (i.e. $x=0.265$ ) ends of tank and at the middle of the free surface (i.e. $x=0$ ).

For non-resonant frequency motion, the numerical solution of sloshing by the proposed method is in a quite acceptable agreement with the reference solution and analytical formulation in terms of elevation of free surface. Figure 5 presents the time history response of free surface elevations at three measurement locations (i.e. $x=-0.265,0$ and $0.265 \mathrm{~m}$ ) that is extended to $20 \mathrm{~s}$. The maximum sloshing amplitude at $x=+0.265 \mathrm{~m}$ is measured as $0.0062 \mathrm{~m}$ from the numerical solution whereas, it is obtained as $0.0067 \mathrm{~m}$ and $0.0064 \mathrm{~m}$ from the analytical formulation and experimental data, respectively. The minimum free surface elevation is obtained as $0.0063 \mathrm{~m}$, $0.0070 \mathrm{~m}$ and $0.0061 \mathrm{~m}$ from experimental, analytical and numerical methods, respectively. In both cases, the numerical results are quite close to the experimental ones. As it is expected, the wave height is nearly zero at the middle of the free surface. 


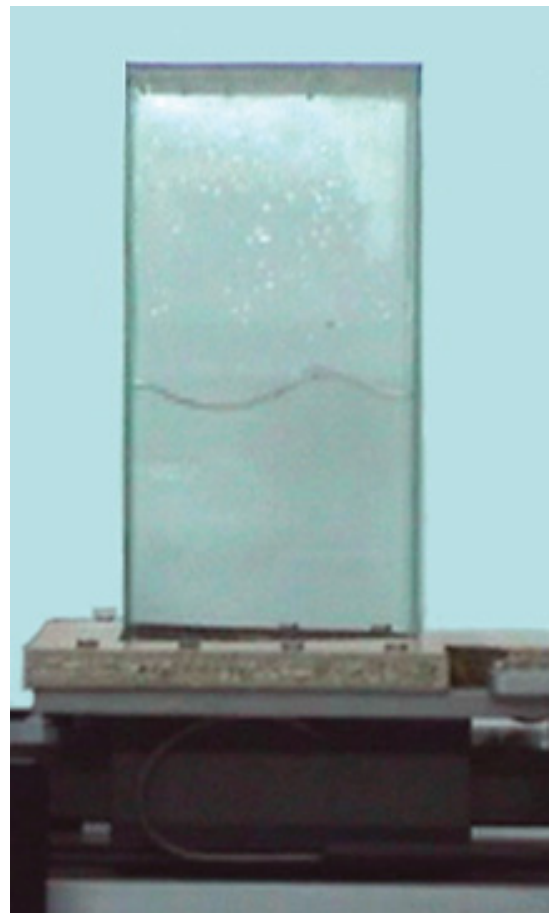

Time $=\quad 0.76$

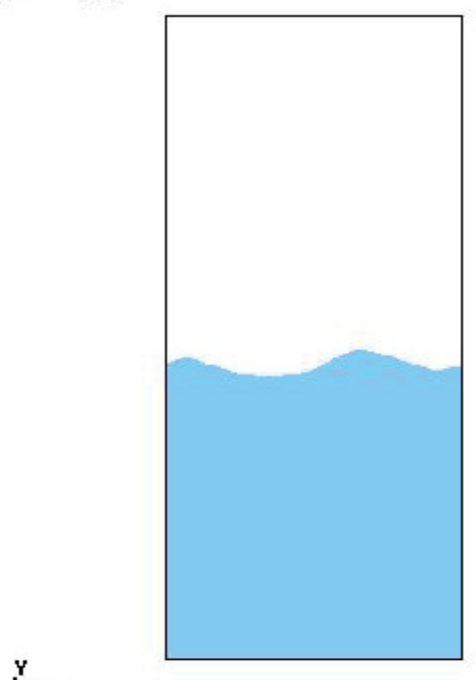

Fig. 4. Free surface profiles of experimental and numerical models under sinusoidal excitation with amplitude $0.005 \mathrm{~m}$ and frequency $3.5 \mathrm{~Hz}$.
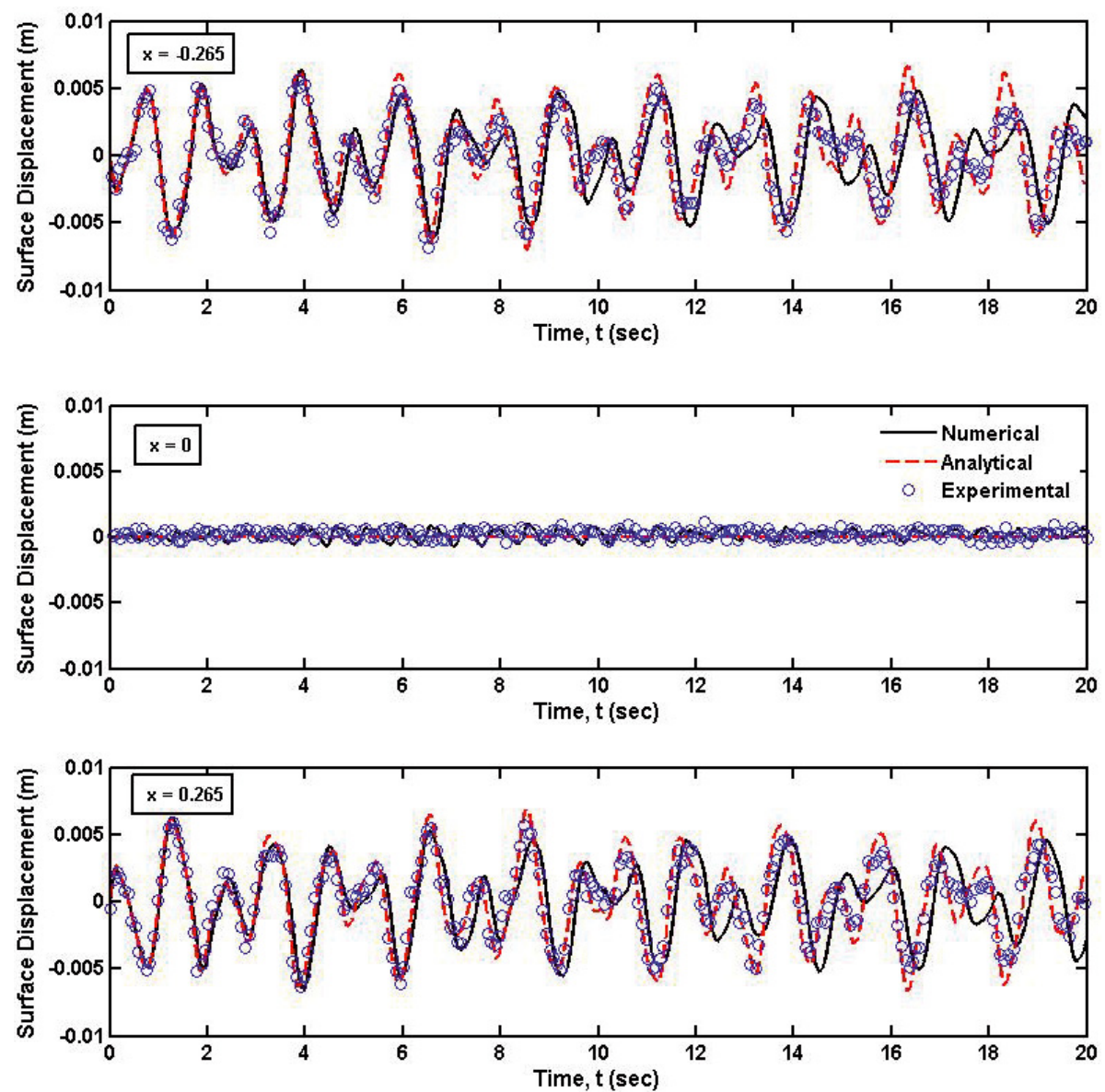

Fig. 5. Comparison of sloshing wave height time histories at three measurement points obtained by numerical and analytical methods and experimental data (non-resonant case). 

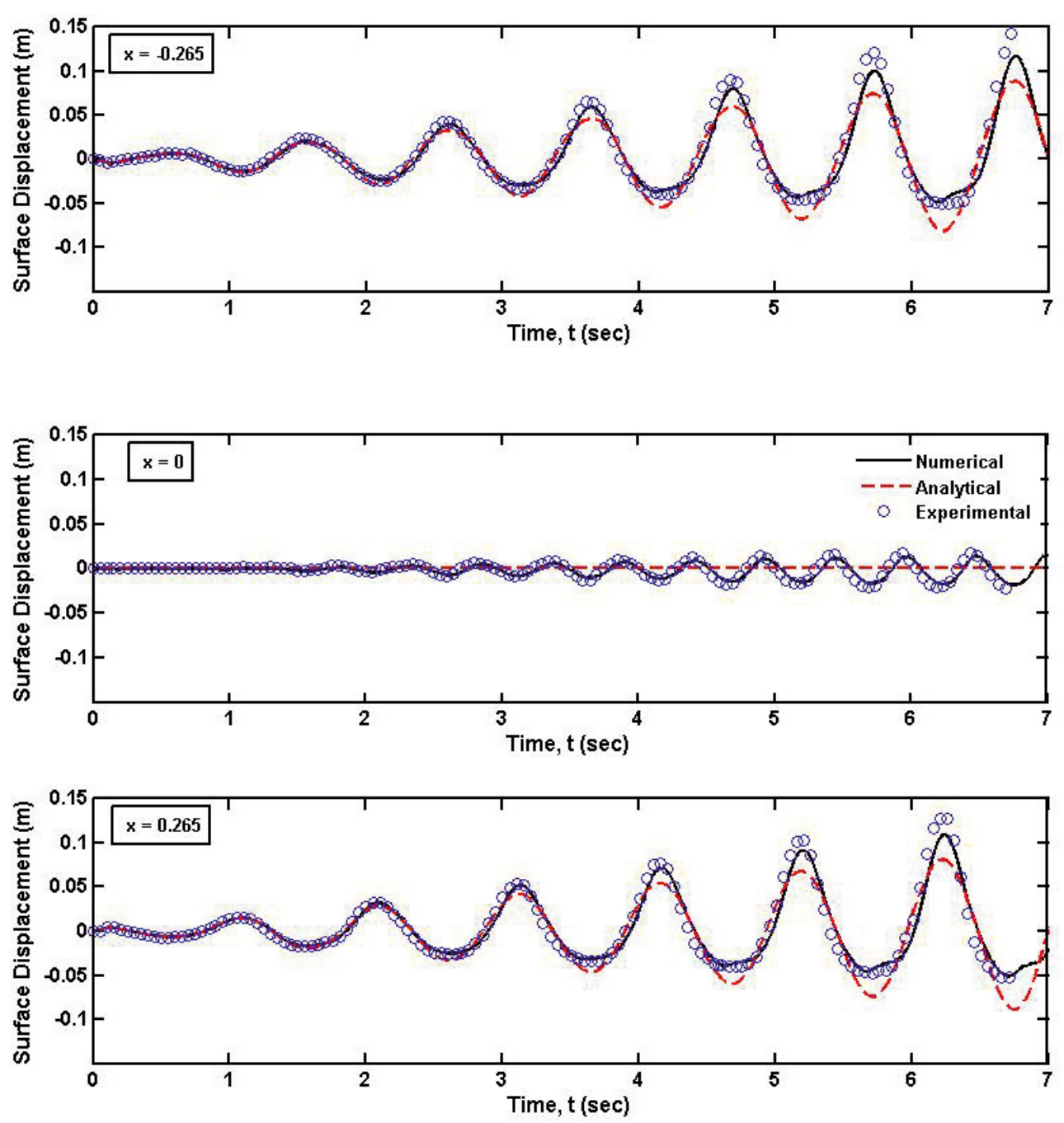

Fig. 6. Comparison of sloshing wave height time histories at three measurement points obtained by numerical and analytical methods and experimental data (resonant case).

For the resonant frequency case, the wave height increases continuously over time for all solution types at the near left and right end of the tank. The comparison of three solution methods shows that analytical study overestimates negative surface amplitudes, whereas it underestimates the positive ones (Fig. 6). Numerical and experimental results are highly consistent in terms of peak level timing, shape and amplitude of sloshing wave. The free surface displacement time histories obtained from numerical and experimental studies show that the positive (upward) sloshing wave amplitudes are always larger than the negative (downward) ones. This phenomenon is a classical indication of a nonlinear behavior of sloshing and caused by suppression effect of the tank base on waves with negative amplitude. Although the gravity effects exist for upward and downward fluid motion, the downward motion of fluid is blocked by the tank bottom. The ratio of positive amplitude to absolute negative amplitude increases as the fluid depth decreases. This phenomenon can not be observed from analytical solution because it is derived under linearized assumptions.
Pressure time histories, including hydrostatic pressure, observed at three different locations which are at the left $(x=-0.285)$ and right $(x=0.285)$ sides of the tank ( $0.01 \mathrm{~m}$ above the base) and at the middle of the tank base are plotted in Figures 7 and 8 for resonant and nonresonant frequency motions, respectively. Since pressure was not measured in the experimental study, only analytical and numerical results are compared. Although hydrostatic pressure field is generated by increasing the gravity gradually at the first one second of the analysis there is a high frequency oscillation region in pressure response at the first $3 \mathrm{~s}$ of the analysis. For non-resonant frequency motion, there is a small phase difference in pressure time histories observed by analytical and numerical methods at the left and right sides of the tank. Pressure measured at the middle of the tank base includes almost only hydrostatic pressure effect. For non-resonant frequency motion, the contribution of hydrodynamic pressure to total pressure response is very small value.

For resonant frequency loading case, nonlinear sloshing action at the free surface causes small amplitude 

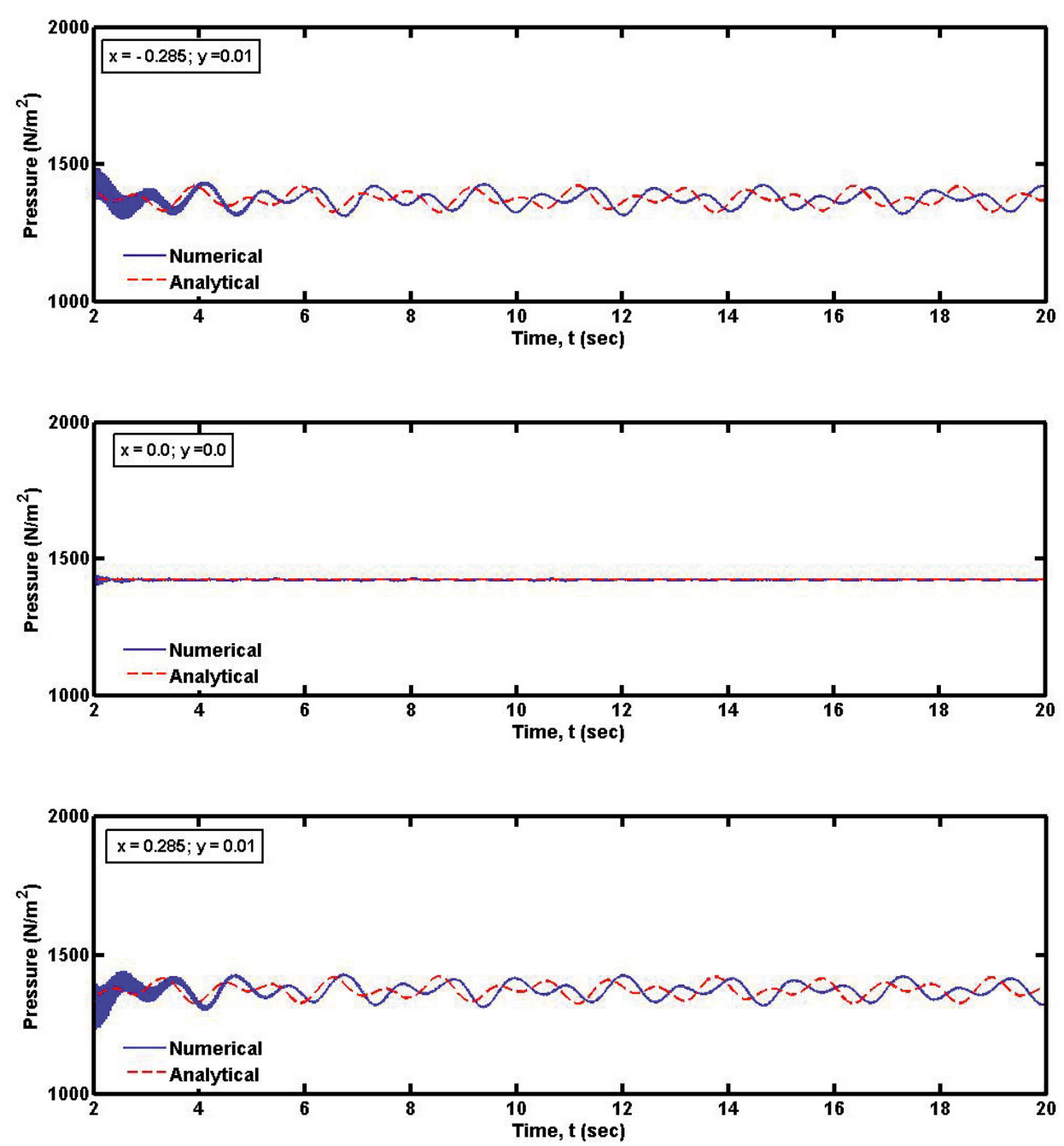

Fig. 7. Comparison of pressure time histories at three locations on the tank wall obtained by numerical and analytical methods (non-resonant case).

oscillations in pressure observed at the middle of the tank base plate, but analytical method reflects only hydrostatic pressure effect at the same point without any oscillation. Pressure response at the two edges of the tank is almost symmetric with respect to vertical axis passing at the middle of the tank. Although peak level timing of pressure time histories obtained by analytical and numerical methods is perfectly consistent, pressure obtained from analytical study at the right and left sides of the tank continuously increases over time in an unbounded manner. However, pressure observed by numerical model oscillates between the same negative and positive values after $10 \mathrm{~s}$. Therefore, it can be concluded that analytical method is not reliable for resonant frequencies due to the unbounded response.

\subsection{Sloshing wave height under recorded earthquake motion}

Liquid sloshing inside a scaled 3D rectangular tank model induced by recorded earthquake excitation is evaluated with presented numerical method and the results are compared with experimental study data presented by Chen et al. [17]. The width, breath and height of the tank are $0.80,0.141$ and $0.20 \mathrm{~m}$, respectively. The tank is filled with water at a height of $0.10 \mathrm{~m}$. The forced excitation of tank is taken as the horizontal (east-west) component of the 1999 Chi-Chi earthquake (Taiwan) recorded at the $\mathrm{CHY} 028$ ground station. The motion is scaled by $1 \%$ and transformed into the ground displacement in order to use as input for nonlinear dynamic time history analysis. The displacement time history of input motion is shown in Figure 9a.

Figure $9 \mathrm{~b}$ represents time history responses of the free surface elevation of the model under consideration, compared with the experimental data, where the elevation is measured at the right end $x=+0.40 \mathrm{~m}$. It can be noticed from the figure that the numerical and experimental models lead to a relatively accurate description of the water sloshing in terms of the displacement of free surface for recorded earthquake motion. Therefore, it can be justified that the presented method is also reliable for the analysis 

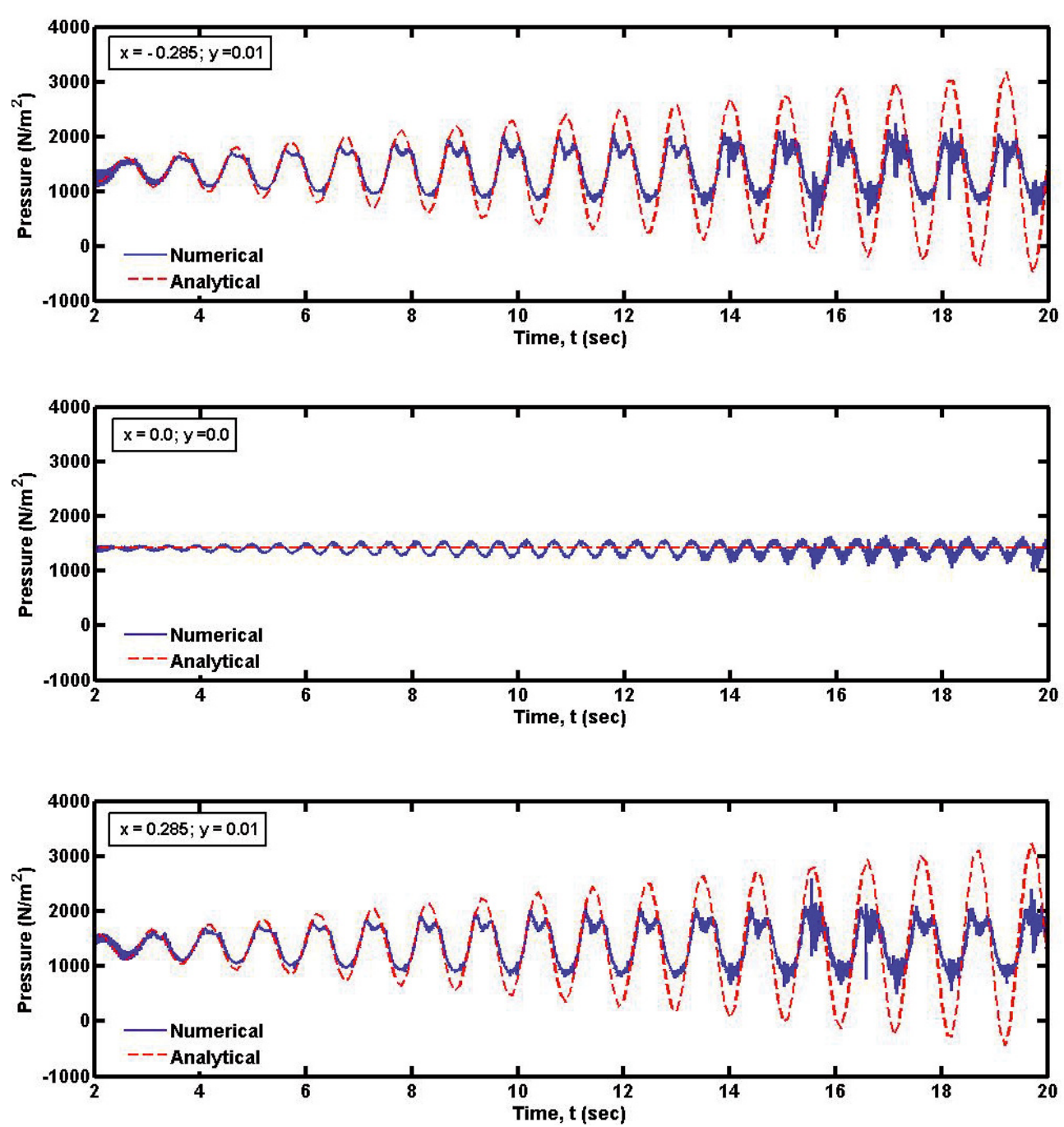

Fig. 8. Comparison of pressure time histories at three locations on the tank wall obtained by numerical and analytical methods (resonant case).

of sloshing inside a tank subjected to earthquake excitation.

\section{Conclusions}

This paper presents a fully non-linear numerical method based on arbitrary Lagrangian Eulerian algorithm of finite-element method to solve the threedimensional fluid-structure interaction problems. In this method, Navier-Stokes equations are employed to represent the motion of the fluid domain. The volume-of-fluid (VOF) method is adopted to track the free surface motion. The momentum advection algorithm is applied to transfer the materials across element boundaries. A second order accurate time marching procedure based on central difference method is used to advance the solution in time. The presented method is utilized to solve the sloshing problems in fixed based tanks. In order to validate the applicability of the numerical algorithm to sloshing problems, simulations on small-scale rigid test models are carried out for different loading cases. As a preliminary step, experimental tests on small scale tank model are carried out and free surface profiles observed from these experiments are compared with the numerical simulations. It is obviously indicated that the free surface profiles obtained from experimental and numerical studies perfectly match each other. In the sequential numerical analyses, time history of sloshing wave elevation is investigated for resonant and non-resonant loading cases. The results of the numerical algorithm are compared with those of analytical method established on potential flow theory and experimental data in terms of sloshing wave elevation. Experimental data are used as reference solution for sloshing problem. For non-resonant frequency motion, the numerical and analytical results agree excellently with experimental data. For resonant case, even though, the linear analytical solution has maximum $50 \%$ difference from the experimental data, the numerical results match very well with the experimental data that exhibit nonlinear wave effects. The pressure time histories at specific locations obtained by numerical method are compared 


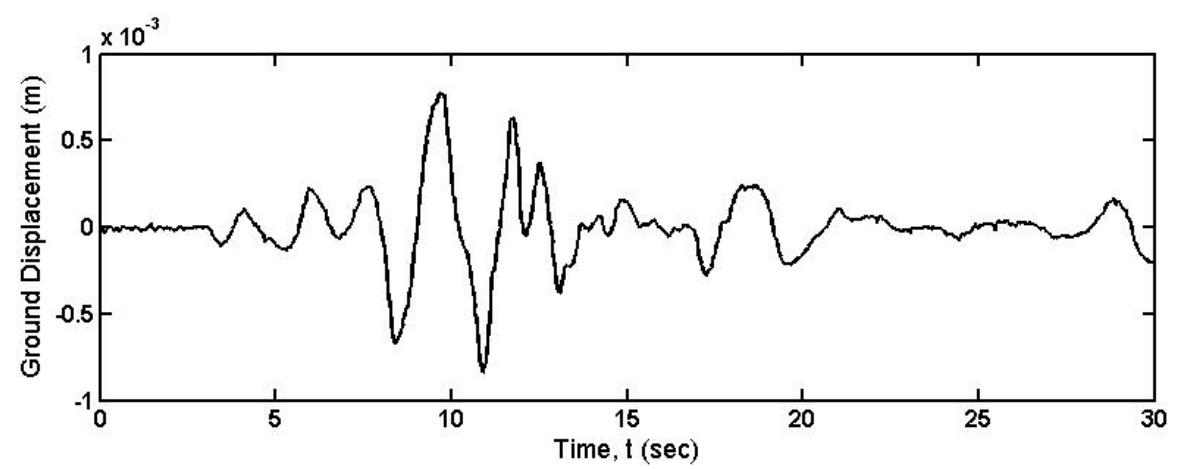

(a)

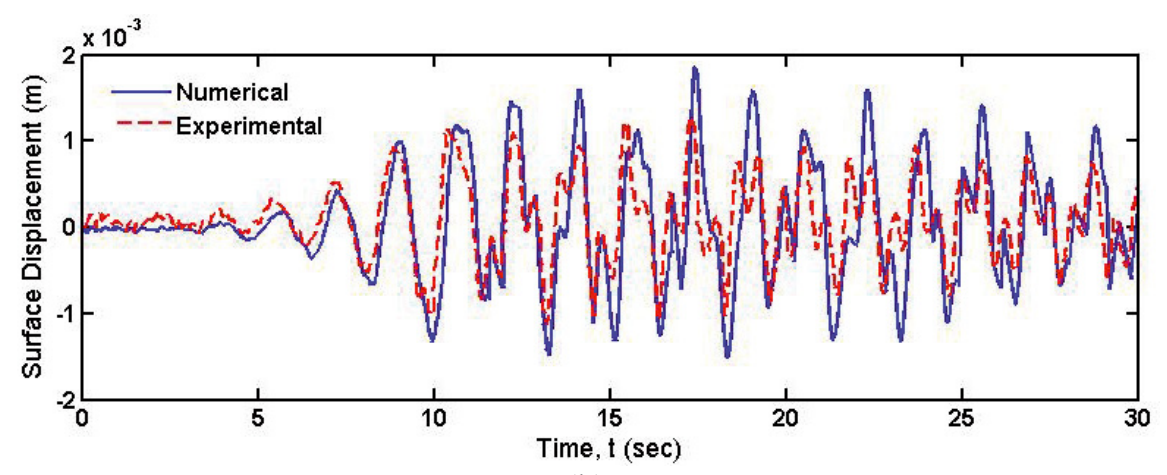

(b)

Fig. 9. Ground displacement of scaled Chi-Chi earthquake (1999) and time history of free surface displacement at the right wall of the tank.

with linear analytical results. Although peak level timing of pressure time histories obtained by analytical and numerical methods is perfectly consistent for resonant frequency loading case, pressure obtained by analytical study at the right and left sides of the tank continuously increase over time in an unbounded manner. However, pressure observed by numerical model oscillates between the same negative and positive values after $10 \mathrm{~s}$. The presented numerical method is also compared well with shaking table test results for recorded earthquake excitation. It can be concluded that the present numerical algorithm is reliable and useful for sloshing problems in practice for every frequency range of external excitation.

Acknowledgements. The authors would like to express their deepest gratitude to Professor Erdal Safak of Kandilli Observatory of Bogazici University for careful review of the manuscript and his valuable recommendations. This work is supported by the Scientific and Technological Research Council of Turkey (TUBITAK-MAG) through contract number 108M607 and by Research Fund of Gebze Institute of Technology under project number 2009-A-25. The first author is granted by Eiffel excellence scholarship of the French Ministry of Foreign and European Affairs.

\section{References}

[1] L.S. Jacobsen, Impulsive hydrodynamics of fluid inside a cylindrical tank and of fluid surrounding a cylindrical pier, Bull. Seismological Soc. Amer. 39 (3) (1949) 189-204
[2] G.W. Housner, Earthquake pressures on fluid containers. 8th Technical Report under Office of Naval Research, California Institute of Technology, Pasadena, California, August, 1954

[3] G.W. Housner, Dynamic pressures on accelerated fluid containers. Bull. Seismological Soc. Amer. 47 (1) (1957) $15-35$

[4] G.W. Housner, The dynamic behavior of water tanks. Bull. Seismological Soc. Amer. 53 (2) (1963) 381-387

[5] A.S. Veletsos, J.Y. Yang, Earthquake response of liquid storage tanks. Advances in Civil Engineering Through Engineering Mechanics, Proceedings of the Engineering Mechanics Division Specialty Conferences, ASCE, Raleigh, North Carolina (1977) 1-24

[6] O.M. Faltinsen, A numerical nonlinear method of sloshing in tanks with two-dimensional flow, J. Ship Res. 22 (1978) 193-202

[7] F.D. Fischera, F.G. Rammerstorferb, A Refined Analysis of Sloshing Effects in Seismically Excited Tanks, Int. J. Press. Vessels Piping 76 (1999) 693-709

[8] K.S. Lay, Seismic Coupled Modeling of Axisymmetric Tanks Containing Liquid, J. Eng. Mech. 119 (1993) 17471761

[9] A. El-Zeiny, Nonlinear Time-Dependent Seismic Response of Unanchored Liquid Storage Tanks, Ph.D. Dissertation, Department of Civil and Environmental Engineering, University of California, Irvine, 1995

[10] B.F. Chen, H.W. Chiang, Complete 2D and Fully Nonlinear Analysis of Ideal Fluid in Tanks, J. Eng. Mech. ASCE 125 (1999) 70-78 
[11] B.F. Chen, Viscous Fluid in Tank under Coupled Surge, Heave, and Pitch Motions, Journal of Waterway, Port, Coastal, and Ocean Engineering ASCE 131 (2005) 239256

[12] M. Souli, A. Ouahsine, L. Lewin, ALE formulation for fluid-structure interaction problems, Comput. Methods Appl. Mech. Eng. 190 (2000) 659-675

[13] M. Souli, J.P. Zolesio, Arbitrary Lagrangian-Eulerian and free surface methods in fluids mechanics, Comput. Methods Appl. Mech. Eng. 191 (2001) 451-466

[14] E. Longatte, Z. Benddjedou, M. Souli, Methods for numerical study of tube bundle vibrations in cross-flows, J. Fluids Struct. 18 (2003) 513-528

[15] E. Longatte, Z. Bendjeddou, M. Souli, Application of Arbitrary Lagrange Euler Formulations to Flow-Induced Vibration problems, J. Press. Vessel Technology 125 (2003) 411-417

[16] N. Aquelet, M. Souli, L. Olovson, Euler Lagrange coupling with damping effects: Application to slamming problems, Comput. Methods Appl. Mech. Eng. 195 (2005) 110-132

[17] Y.H. Chen, W.S. Hwang, C.H. Ko, Sloshing Behaviours of Rectangular and Cylindrical Liquid Tanks Subjected to Harmonic and Seismic Excitations, Earthquake Engineering and Structural Dynamics 36 (2007) 17011717

[18] D. Liu, P. Lin, A numerical study of three-dimensional liquid sloshing in tanks, J. Comput. Phys. 227 (2008) 3921-3939

[19] S. Mitra, P.P. Upadhyay, K.P. Sinhamahapatra, Slosh Dynamics of Inviscid Fluids in Two-Dimensional Tanks of Various Geometry Using Finite Element Method, Int. J. Num. Methods Fluids 56 (2008) 1625-1651

[20] D.D. Kana, Seismic Response of Flexible Cylindrical Liquid Storage Tanks, Nuclear Engineering and Design 52 (1979) 185-199

[21] G.C. Manos, Dynamic response of a broad storage tank model under a variety of simulated earthquake motions. Proc. 3rd U.S. Nat. Conf. on Earthquake Engrg., Earthquake Engineering Research Institute, E1 Cerrito, Calif., 1986, pp. 2131-2142

[22] Tanaka, Motoaki, Sakurai, Ishida, Tazuke, Akiyama, Kobayashi and Chiba, Proving Test of Analysis Method on Nonlinear Response of Cylindrical Storage Tank Under Severe Earthquakes, Proceedings of 12th World Conference on Earthquake Engineering (12 WCEE), Auckland, New Zealand, 2000

[23] R.A. Ibrahim, Liquid Sloshing Dynamics: Theory and Applications, Cambridge University Press, New York, USA, 2005

[24] T. Belytschko, W.K. Liu, B. Moran, Nonlinear finite elements for continua and structures, Wiley, New York, 2000

[25] D.J. Benson, Computational Methods in Lagrangian and Eulerian Hydrocodes, Comput. Methods Appl. Mech. Eng. 99 (1992) 235-394
[26] T.J.R. Hughes, W.K. Liu, T.K. Zimmerman, Lagrangian Eulerian finite element formulation for viscous flows, J. Comput. Methods Appl. Mech. Eng. 29 (1981) 329-349

[27] S. Ghosh, N. Kikuchi, An arbitrary Lagrangian-Eulerian finite element method for large deformation analysis of elastic-viscoplastic solid, Comput. Meth. Appl. Mech. Engng. 86 (1991) 127-188

[28] J. Donea, Arbitrary Lagrangian-Eulerian Finite Element Methods, Computational methods four Transient Analysis, T. Belytschko, T.J.R. Hughes (eds.) Elsevier Sciences Publishers, B.V. 1983, pp. 473-513

[29] D.J. Benson, A mixture theory for contact in multimaterial eulerian formulations, Comput. Meth. Appl. Mech. Eng. 140 (1997) 59-86

[30] D.P. Flanagan, T. Belytschko, A Uniform Strain Hexahedron and Quadrilateral and Orthogonal Hourglass Control, Int. J. Numer. Meths, Eng. 17 (1981) 679-706

[31] F. Erchiqui, M. Souli, R.B. Yedder, Nonisothermal FiniteElement Analysis of Thermoforming of Polyethylene Terephthalate Sheet: Incomplete Effect of the Forming Stage, Polymer Engineering and Science (2007) 21292144

[32] C.W. Hirt, B.D. Nichols, Volume of fluid (VOF) method for the dynamics of free boundaries. J. Comput. Phys. 39 (1981) 201-225

[33] F.H. Halow, J.E. Welch, Numerical calculation of timedependent viscous incompressible flow of fluid with a free surface, Phys. Fluids 12 (1965) 2182

[34] J.A. Viecelli, A method for including arbitrary external boundaries in the MAC incompressible fluid computing technique, J. Comput. Phys. 4 (1969) 543

[35] D.L. Young, Time-dependent multi-material flow with large fluid distortion, Numerical Methods for Fluids Dynamics, K.W. Morton, M.J. Baines (eds.), Academic Press, New-York, 1982

[36] N. Aquelet, M. Souli, J. Gabrys, L. Olovsson, A new ALE formulation for sloshing analysis, Struct. Eng. Mech. 16 (2003) 423-440

[37] A. Alia, M. Souli, High explosive simulation using multimaterial formulations, Appl. Therm. Eng. 26 (2006) 1032-1042

[38] P.R. Woodward, P. Collela, The numerical simulation of two-dimensional fluid flow with strong shocks, Lawrence Livermore National Laboratory, UCRL-86952, 1982

[39] B. Van Leer, Towards the Ultimate Conservative Difference Scheme. IV. A New Approach to Numerical Convection, J. Comput. Phys. 23 (1977) 276-299

[40] D.J. Benson, Eulerian finite element methods for the micromechanics of heterogenous materials: Dynamics prioritization of material interfaces, Comput. Methods Appl. Mech. Eng. 150 (1998) 343-360

[41] A.A. Amsden, C.W. Hirt, YAQUI: An Arbitrary Lagrangian-Eulerian Computer Program for Fluid Flow at All Speeds, Los Alamos Scientific Laboratory, LA5100,1973 